Comment. Math. Helv. 75 (2000) 216-230

(C) 2000 Birkhäuser Verlag, Basel

$0010-2571 / 00 / 020216-15 \$ 1.50+0.20 / 0$

Commentarii Mathematici Helvetici

\title{
Walls for Gieseker semistability and the Mumford-Thaddeus principle for moduli spaces of sheaves over higher dimensional bases
}

\author{
Alexander Schmitt
}

\begin{abstract}
Let $X$ be a projective manifold over $\mathbb{C}$. Fix two ample line bundles $H_{0}$ and $H_{1}$ on $X$. It is the aim of this note to study the variation of the moduli spaces of Gieseker semistable sheaves for polarizations lying in the cone spanned by $H_{0}$ and $H_{1}$. We attempt a new definition of walls which naturally describes the behaviour of Gieseker semistability. By means of an example, we establish the possibility of non-rational walls which is a substantially new phenomenon compared to the surface case. Using the approach by Ellingsrud and Göttsche via parabolic sheaves, we were able to show that the moduli spaces undergo a sequence of GIT flips while passing a rational wall. We hope that our results will be helpful in the study of the birational geometry of moduli spaces over higher dimensional bases.
\end{abstract}

Mathematics Subject Classification (2000). 14J60, 14D20.

Keywords. (Semi)stable sheaf, moduli space, polarization, flip, Riemann-Roch, parabolic sheaf.

\section{Introduction}

Fix an $n$-dimensional smooth projective manifold $X$ over the complex numbers as well as a function $p: \operatorname{Num}(X) \longrightarrow \mathbb{Z}$, called Hilbert form. Define $N_{\mathbb{Q}}^{1}(X):=$ $\operatorname{Num}(X) \otimes_{\mathbb{Z}} \mathbb{Q}$ and similarly $N_{\mathbb{R}}^{1}(X)$, and finally let $\operatorname{Amp}_{\mathbb{Q}}(X)$ and $\operatorname{Amp}_{\mathbb{R}}(X)$ be the cones in $N_{\mathbb{Q}}^{1}(X)$ and $N_{\mathbb{R}}^{1}(X)$, resp., spanned by the classes of ample line bundles. Assuming that $H$ is the class of an ample line bundle, we define $P_{H}(\mathcal{E})$ as the polynomial such that $P_{H}(\mathcal{E})(n)=\chi\left(\mathcal{E} \otimes H^{\otimes n}\right)$ for any natural number $n$. The sheaf $\mathcal{E}$ is then called Gieseker $H$-(semi)stable (or just $H$-(semi)stable) if and only if every non-zero proper subsheaf $\mathcal{F}$ of $\mathcal{E}$ satisfies $P_{H}(\mathcal{F}) / \mathrm{rk} \mathcal{F} \quad(\leq) \quad P_{H}(\mathcal{E}) / \mathrm{rk} \mathcal{E}$. There is a projective moduli space $\mathcal{M}_{H}:=\mathcal{M}_{H}(p)$ of S-equivalence classes of Gieseker $H$-semistable torsion free coherent sheaves $\mathcal{E}$ with Hilbert form $p$, i.e., $p([D])=\chi\left(\mathcal{E} \otimes \mathcal{O}_{X}(D)\right)$ for all $[D] \in \operatorname{Num}(X)$. Note that this determines the rank of $\mathcal{E}$, henceforth denoted by $r$, the numerical equivalence class of $c_{1} \mathcal{E}$, henceforth denoted by $c_{1}$, and $c_{2} \mathcal{E}$ as a linear form on the subvectorspace of $H^{2 n-4}(X, \mathbb{Q})$ spanned by $(n-2)$-fold intersections of divisors, as such it is called $c_{2}$. By its 
very definition, the space $\mathcal{M}_{H}$ depends on the chosen polarization, and it is an interesting and important problem to compare $\mathcal{M}_{H_{0}}$ to $\mathcal{M}_{H_{1}}$ for different polarizations $H_{0}$ and $H_{1} \in \mathrm{Amp}_{\mathbb{Q}}(X)$. For surfaces, this problem has been thoroughly studied. A brief discussion of this topic and appropriate references can be found in [5]. The most general result in this direction has been obtained in [7] where it is shown that the moduli spaces are related by a sequence of GIT flips. A similar result can be obtained using moduli spaces of parabolic sheaves as mentioned in the paper [3]. In this note we aim at a generalization of the results of [7] to higher dimensions, using the approach of [3]. However, there arise new problems due to the appearance of walls which do not lie in $N_{\mathbb{Q}}^{1}(X)$. Our result is summarized in the following

Main Theorem. Given two polarizations $H_{0}$ and $H_{1}$, there is a finite subset $w$ of $\Delta:=\left\{(1-\lambda) H_{0}+\lambda H_{1} \mid \lambda \in[0,1]\right\}$ such that the notion of Gieseker (semi)stability remains constant within each connected component of $\Delta \backslash w$. If the polarization passes through a wall of $w \cap N_{\mathbb{Q}}^{1}(X)$, then the moduli spaces undergo a sequence of $\mathbb{C}^{*}$-flips.

In the case of crossing a real wall, one cannot expect such a result, because it would yield an algebro geometric construction of a moduli space of Gieseker semistable sheaves w.r.t. a real polarization which seems most unlikely in my eyes. However, in this case, some suitable fibre spaces over the moduli spaces can be obtained by a sequence of $\mathbb{C}^{*}$-flips from the same Quot scheme. This will be explained in Section 3.

In general, the hope is that $\mathcal{M}_{H_{0}}$ and $\mathcal{M}_{H_{1}}$ will be - under suitable assumptions - birational to each other, although other results indicate that moduli spaces over higher dimensional bases are not at all well-behaved, e.g., they can have arbitrarily many components ([2], [1]). The flips between the moduli spaces can be very helpful in this context. In fact, one should be able to obtain quite explicit descriptions of the exceptional sets of the flips. Then, one is left with estimating the dimension of these exceptional sets, and this might be the hard part.

In the case of crossing a rational wall, our construction gives the following: There is a quasi-projective scheme $\mathfrak{X}$, an ample line bundle $\mathfrak{L}$ on $\mathfrak{X}$, and a $\mathbb{C}^{*}$ action on $\mathfrak{X}$ together with two linearizations $\sigma_{0}$ and $\sigma_{1}$ of this action in $\mathfrak{L}$ such that $\mathfrak{X} / / \sigma_{0,1} \mathbb{C}^{*}=\mathcal{M}_{H_{0,1}}$. Let $\mathfrak{X}_{i}, i=1, \ldots, t$, be the irreducible components of $\mathfrak{X}$. Since $\mathbb{C}^{*}$ is irreducible, the action preserves those components. So, the $\mathcal{M}_{0,1}^{i}$ will be the irreducible components of $\mathcal{M}_{H_{0,1}}, i=1, \ldots, t$. By general properties of $\mathbb{C}^{*}$-actions (e.g. [11], [8]) one gets

Corollary. Under the above hypotheses, if for $i_{0} \in\{1, \ldots, t\}$ both $\mathcal{M}_{0}^{i_{0}}$ and $\mathcal{M}_{1}^{i_{0}}$ are non-empty, then they are birationally equivalent. 


\section{Preparations}

\subsection{Walls for slope semistability}

For technical reasons, we will have to consider the notion of slope semistability for all $H \in \operatorname{Amp}_{\mathbb{R}}(X)$. So, let us fix such an $H$. For a torsion free coherent sheaf $\mathcal{E}$, define its $H$-slope as $\mu_{H} \mathcal{E}:=c_{1} \mathcal{E} \cdot H^{n-1} / \mathrm{rk} \mathcal{E}$ and call $\mathcal{E}$ slope $H$-(semi)stable if $\mu_{H} \mathcal{F}(\leq) \mu_{H} \mathcal{E}$ for any non-zero proper subsheaf $\mathcal{F}$ of $\mathcal{E}$.

Example 1.1.1. Let $X \subset \mathbb{P}_{2} \times \mathbb{P}_{2}$ be a smooth hypersurface in $|\mathcal{O}(1,1)|$. The nef cone of $X$ is spanned by $H_{0}:=\pi_{1}^{*} \mathcal{O}_{\mathbb{P}_{2}}(1)$ and $H_{1}:=\pi_{2}^{*} \mathcal{O}_{\mathbb{P}_{2}}(1)$. Set $H_{\lambda}=$ $(1-\lambda) H_{0}+\lambda H_{1}$. We have $H_{0}^{3}=0=H_{1}^{3}$ and $H_{0}^{2} \cdot H_{1}=1=H_{0} \cdot H_{1}^{2}$. Define $E:=\mathcal{O}(2,-1) \oplus \mathcal{O}(-2,1)$. This bundle will be slope $H_{\lambda}$-semistable if and only if

$$
0=\mathcal{O}(2,-1) \cdot H_{\lambda}^{2}=-\lambda^{2}+4 \lambda-1 .
$$

This equation has the (irrational) solutions $\lambda_{ \pm}:=2 \pm \sqrt{3}$. Note that $\lambda_{-}$gives a real class in the ample cone. Hence, $E$ is semistable only with respect to a single real class! Thus, the study of sheaves which are slope semistable w.r.t. a real class cannot necessesarily be reduced to the study of vector bundles which are slope semistable for some rational class.

Fix two polarizations $H_{0}$ and $H_{1}$ in $\operatorname{Amp}_{\mathbb{Q}}(X)$ and denote the line segment joining them by $\Delta$. In this section, $H_{\lambda}$ stands for the polarization $(1-\lambda) H_{0}+\lambda H_{1}$, $\lambda \in[0,1]$. We are interested in the family $\mathfrak{F}(\Delta)$ of isomorphy classes of torsion free coherent sheaves $\mathcal{E}$ with Hilbert form $p$ for which there exists a rational polarization $H \in \Delta \cap N_{\mathbb{Q}}^{1}(X)$ w.r.t. which $\mathcal{E}$ is slope semistable.

For any sheaf $\mathcal{E}$ and any non-zero proper subsheaf $\mathcal{F} \subset \mathcal{E}$ define $\xi_{\mathcal{F}, \mathcal{E}}:=$ $\left[c_{1} \mathcal{F} / \mathrm{rk} \mathcal{F}-c_{1} / r\right]$. We begin with the following observation.

Lemma 1.1.2. Let $\lambda_{0} \in[0,1) \cap \mathbb{Q}$ and $\lambda_{1} \in\left(\lambda_{0}, 1\right)$. Denote the family of isomorphy classes of slope $H_{\lambda_{0}}$-semistable torsion free coherent sheaves with Hilbert form $p$ by $\mathfrak{F}\left(H_{\lambda_{0}}\right)$. Then there is a constant $C$ such that for any $\mathcal{E}$ with $[\mathcal{E}] \in$ $\mathfrak{F}\left(H_{\lambda_{0}}\right)$ and any non-zero proper subsheaf $\mathcal{F}$ of $\mathcal{E}$ the condition $\xi_{\mathcal{F}, \mathcal{E}} \cdot H_{\lambda_{0}}^{n-1}<C$ implies $\xi_{\mathcal{F}, \mathcal{E}} \cdot H_{\lambda}^{n-1}<0$ for all $\lambda \in\left[\lambda_{0}, \lambda_{1}\right]$.

Proof. We may assume $\lambda_{0}=0$. Then $H_{\lambda}^{n-1}=\sum_{i=0}^{n-1}\left(\begin{array}{c}n-1 \\ i\end{array}\right)(1-\lambda)^{i} \lambda^{n-i-1} H_{0}^{i} H_{1}^{n-1-i}$. Since $\mathfrak{F}\left(H_{0}\right)$ is a bounded family, there are constants $K_{0}, \ldots, K_{n-2}$ such that $\xi_{\mathcal{F}, \mathcal{E}} \cdot H_{0}^{i} H_{1}^{n-1-i} \leq K_{i}, i=0, \ldots, n-2$, for all $\mathcal{E}$ with $[\mathcal{E}] \in \mathfrak{F}\left(H_{0}\right)$ and all subsheaves $0 \neq \mathcal{F} \subset \mathcal{E}$. Setting

$$
K:=\max \left\{\sum_{i=0}^{n-2}\left(\begin{array}{c}
n-1 \\
i
\end{array}\right)(1-\lambda)^{i} \lambda^{n-i-1} K_{i} \mid \lambda \in[0,1]\right\},
$$


we conclude that, for $\lambda \in\left[0, \lambda_{1}\right], \mathcal{E}$ with $[\mathcal{E}] \in \mathfrak{F}\left(H_{0}\right)$, and all subsheaves $0 \neq \mathcal{F} \subset \mathcal{E}$,

$$
0 \leq \xi_{\mathcal{F}, \mathcal{E}} \cdot H_{\lambda}^{n-1} \leq(1-\lambda)^{n-1} \xi_{\mathcal{F}, \mathcal{E}} \cdot H_{0}^{n-1}+K \leq\left(1-\lambda_{1}\right)^{n-1} \xi_{\mathcal{F}, \mathcal{E}} \cdot H_{0}^{n-1}+K
$$

implies $\xi_{\mathcal{F}, \mathcal{E}} \cdot H_{0}^{n-1} \geq-K /\left(1-\lambda_{1}\right)^{n-1}$, and we are done.

As important consequence, we note

Proposition 1.1.3. Let $\mathcal{E}$ be a torsion free coherent sheaf such that $[\mathcal{E}] \in \mathfrak{F}(\Delta)$. Suppose that $\mathcal{E}$ is slope semistable w.r.t. $H_{\lambda_{0}}$ with $\lambda_{0} \neq 1$ and that for any subsheaf $\mathcal{F} \subset \mathcal{E}$ there is an open neighborhood $U \subset[0,1]$ of $\lambda_{0}$, such that $\xi_{\mathcal{F}, \mathcal{E} \cdot H_{\lambda}^{n-1} \leq 0}$ for all $\lambda \in U$. Then

- either $\mathcal{E}$ is slope $H_{\lambda}$-semistable for every $\lambda \in\left[\lambda_{0}, 1\right]$,

- or there exists a number $\lambda_{+}>\lambda_{0}$ such that

1. $\mathcal{E}$ is slope $H_{\lambda}$-semistable for every $\lambda \in\left[\lambda_{0}, \lambda_{+}\right]$,

2. there exists a saturated non-zero proper subsheaf $\mathcal{F}_{+} \subset \mathcal{E}$ with $\mu_{H_{\lambda_{+}}} \mathcal{F}_{+}=$ $\mu_{H_{\lambda_{+}}} \mathcal{E}$ such that

$$
\left(\left(\operatorname{rk} \mathcal{F}_{+}-1\right) c_{1}^{2} \mathcal{F}_{+}-2 \operatorname{rk} \mathcal{F}_{+} c_{2} \mathcal{F}_{+}\right) \cdot H_{\lambda_{+}}^{n-2} \leq 0
$$

and, for $\mathcal{G}_{+}:=\mathcal{E} / \mathcal{F}_{+}$,

$$
\left(\left(\operatorname{rk} \mathcal{G}_{+}-1\right) c_{1}^{2} \mathcal{G}_{+}-2 \operatorname{rk} \mathcal{G}_{+} c_{2} \mathcal{G}_{+}\right) \cdot H_{\lambda_{+}}^{n-2} \leq 0
$$

3. $\mathcal{E}$ is not slope $H_{\lambda}$-semistable for $\lambda>\lambda_{+}$close enough.

Remark 1.1.4. i) Likewise, one can construct under the assumption $\lambda_{0} \neq 0$ a number $\lambda_{-}<\lambda_{0}$ and a subsheaf $\mathcal{F}_{-}$with the respective properties.

ii) The need for this proposition arises from the fact that I don't know if the Bogomolov inequality continues to hold for real polarizations.

Proof. We may suppose that $\mathcal{E}$ is not slope $H_{\lambda_{1}}$-semistable for some rational $\lambda_{1}>\lambda_{0}$. If a subsheaf $\mathcal{F}$ slope desemistabilizes $\mathcal{E}$ for some $H_{\lambda}$ with $\lambda \in\left[\lambda_{0}, \lambda_{1}\right]$, then we must have $\xi_{\mathcal{F}, \mathcal{E}} \cdot H_{\lambda_{0}}^{n-1} \geq C$, by Lemma 1.1.2. The set $\mathfrak{C}$ of saturated subsheaves $\mathcal{F}$ of $\mathcal{E}$ with $\xi_{\mathcal{F}, \mathcal{E}} \cdot H_{\lambda_{0}}^{n-1} \geq C$ is bounded ([5], Lem. 1.7.9). In particular, there are only finitely many elements $\xi$ in $\left(1 / r\right.$ !) $\operatorname{Num}(X)$ of the form $\xi_{\mathcal{F}, \mathcal{E}}$ for which there is a $\lambda \in\left[\lambda_{0}, \lambda_{1}\right]$ with $\xi \cdot H_{\lambda}^{n-1} \geq 0$. Denote these elements by $\xi_{1}, \ldots, \xi_{\nu}$ and set $f_{i}(\lambda):=\xi_{i} \cdot H_{\lambda}^{n-1}$. Let $\lambda_{+}$be the smallest number in $\left(\lambda_{0}, \lambda_{1}\right]$ at which one of the polynomial functions $f_{i}(\lambda)$ undergoes a change of sign. Then, by construction, $\mathcal{E}$ is slope semistable for all $H_{\lambda}$ with $\lambda \in\left[\lambda_{0}, \lambda_{+}\right)$, properly slope $H_{\lambda_{+}}$-semistable, and slope unstable for values $\lambda>\lambda_{+}$, close enough.

Moreover, for every inclusion $0 \subset \mathcal{F}_{1} \subset \mathcal{F}_{2} \subset \mathcal{E}$ occuring among subsheaves

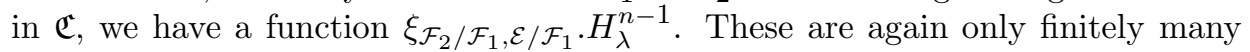


functions. Call them $g_{1}(\lambda), \ldots, g_{\mu}(\lambda)$. We can now choose a number $\lambda^{\prime}$ close to $\lambda_{+}$ in such a way that none of the functions $f_{i}(\lambda), f_{i}(\lambda)-f_{j}(\lambda), i, j=1, \ldots, \nu$, and $g_{i}(\lambda), g_{i}(\lambda)-g_{j}(\lambda), i, j=1, \ldots, \mu$, changes its sign in $\left(\lambda_{+}, \lambda^{\prime}\right)$. We let

$$
0 \subset \mathcal{F}_{1} \subset \cdots \subset \mathcal{F}_{t} \subset \mathcal{E}
$$

be the slope Harder-Narasimhan filtration of $\mathcal{E}$ w.r.t. the polarization $H_{\lambda^{\prime}}$. One has $\mu_{H_{\lambda^{\prime}}} \mathcal{F}_{i}>\mu_{H_{\lambda}} \mathcal{E}$ for $i=1, \ldots, t$, so that the functions $\xi_{\mathcal{F}_{i}, \mathcal{E}} \cdot H_{\lambda}^{n-1}$ are among the functions $f_{i}(\lambda), i=1, \ldots, \nu$. By our choice of $\lambda^{\prime}$, the above filtration is also the slope Harder-Narasimhan filtration of $\mathcal{E}$ w.r.t. to all the polarizations $H_{\lambda}$, $\lambda \in\left(\lambda_{+}, \lambda^{\prime}\right)$. We choose $\mathcal{F}_{+}:=\mathcal{F}_{t}$. Then, $\mathcal{G}_{+}:=\mathcal{E} / \mathcal{F}_{+}$is slope $H_{\lambda}$-semistable w.r.t. all polarizations $\lambda \in\left[\lambda_{+}, \lambda^{\prime}\right]$, and the Bogomolov Theorem ([5], Thm. 7.3.1) implies

$$
\left(\left(\mathrm{rk} \mathcal{G}_{+}-1\right) c_{1}^{2} \mathcal{G}_{+}-2 \mathrm{rk} \mathcal{G}_{+} c_{2} \mathcal{G}_{+}\right) \cdot H_{\lambda}^{n-2} \leq 0
$$

for all $\lambda \in\left[\lambda_{+}, \lambda^{\prime}\right]$. We claim that we also have

$$
\left(\left(\operatorname{rk} \mathcal{F}_{i}-1\right) c_{1}^{2} \mathcal{F}_{i}-2 \operatorname{rk} \mathcal{F}_{i} c_{2} \mathcal{F}_{i}\right) \cdot H_{\lambda_{+}}^{n-2} \leq 0
$$

for $i=1, \ldots, t$. For $\mathcal{F}_{1}$ it follows from the fact that this sheaf is slope $H_{\lambda}$-semistable for all $\lambda \in\left[\lambda_{+}, \lambda^{\prime}\right]$. Suppose now that we have established the above inequality for $\mathcal{F}_{i}$. Write

$$
D(\mathcal{F}):=\left((\operatorname{rk} \mathcal{F}-1) c_{1}^{2} \mathcal{F}-2 \operatorname{rk} \mathcal{F} c_{2} \mathcal{F}\right)
$$

for every coherent sheaf $\mathcal{F}$. We have an exact sequence $0 \longrightarrow \mathcal{F}_{i} \longrightarrow \mathcal{F}_{i+1} \longrightarrow$ $\mathcal{F}_{i+1} / \mathcal{F}_{i} \longrightarrow 0$. The sheaf $\mathcal{F}_{i+1} / \mathcal{F}_{i}$ is again slope $H_{\lambda^{-}}$-semistable for all $\lambda \in\left[\lambda_{+}, \lambda^{\prime}\right]$, so that $D\left(\mathcal{F}_{i+1} / \mathcal{F}_{i}\right) \cdot H_{\lambda}^{n-2} \leq 0$ for all $\lambda \in\left[\lambda_{+}, \lambda^{\prime}\right]$. One has the equality

$$
\frac{D\left(\mathcal{F}_{i+1}\right)}{\operatorname{rk} \mathcal{F}_{i+1}}=\frac{D\left(\mathcal{F}_{i}\right)}{\operatorname{rk} \mathcal{F}_{i}}+\frac{D\left(\mathcal{F}_{i+1} / \mathcal{F}_{i}\right)}{\operatorname{rk} \mathcal{F}_{i+1} / \mathcal{F}_{i}}+\frac{\operatorname{rk} \mathcal{F}_{i} \mathrm{rk} \mathcal{F}_{i+1}}{\operatorname{rk} \mathcal{F}_{i+1} / \mathcal{F}_{i}} \xi_{\mathcal{F}_{i}, \mathcal{F}_{i+1}}^{2} .
$$

Since $\xi_{\mathcal{F}_{i}, \mathcal{F}_{i+1}} \cdot H_{\lambda_{+}}^{n-1}=0$, the Hodge-Riemann bilinear relations ([4], p. 123) for the Kähler class $H_{\lambda_{+}}$imply $\xi_{\mathcal{F}_{i}, \mathcal{F}_{i+1}}^{2} \cdot H_{\lambda_{+}}^{n-2} \leq 0$, and we are done.

Example 1.1.5. This time, we consider a smooth hypersurface $X \subset \mathbb{P}_{2} \times \mathbb{P}_{2}$ in the linear system $|\mathcal{O}(3,3)|$. Using notations analogous to those in Example 1.1.1, we have generators $H_{0}$ and $H_{1}$ of the nef cone of $X$ with $H_{0}^{3}=0=H_{1}^{3}$ and $H_{0}^{2} \cdot H_{1}=3=H_{0} \cdot H_{1}^{2}$. The space $X$ is a Calabi-Yau threefold with $c_{2}(X)=$ $3 H_{0}^{2}+3 H_{1}^{2}+9 H_{0} H_{1}$. First, we check that there is a non-split extension

$$
0 \longrightarrow \mathcal{O}_{X}(3,0) \longrightarrow E \longrightarrow \mathcal{O}_{X}(0,1) \longrightarrow 0 .
$$

Such extensions are parametrized by

$$
\operatorname{Ext}^{1}\left(\mathcal{O}_{X}(0,1), \mathcal{O}_{X}(3,0)\right)=H^{1}\left(\mathcal{O}_{X}(3,-1)\right)
$$


Observe $h^{0}\left(\mathcal{O}_{X}(3,-1)\right)=0=h^{0}\left(\mathcal{O}_{X}(-3,1)\right)=h^{3}\left(\mathcal{O}_{X}(3,-1)\right)$, so that RiemannRoch gives

$-h^{1}(\mathcal{O}(3,-1)) \leq(1 / 6)\left(3 H_{0}-H_{1}\right)^{3}+(1 / 12)\left(3 H_{0}-H_{1}\right) \cdot\left(3 H_{0}^{2}+3 H_{1}^{2}+9 H_{0} H_{1}\right)=-3$.

Besides subsheaves of $\mathcal{O}(3,0), E$ could have subsheaves of the form $\mathcal{O}(-k, 1)$ with $k \geq 1$, because the extension does not split. Subsheaves of the latter form do not destabilize if $\xi_{\mathcal{O}(-1,1), E} \cdot H_{\lambda}^{2}<0$ where $\xi_{\mathcal{O}(-1,1), E}=-(5 / 2) H_{0}+(1 / 2) H_{1}$. One checks that this is fulfilled for all $\lambda>\lambda^{*}:=(5 / 4)-(\sqrt{21} / 4)$. Thus, for $\lambda>\lambda^{*}$, the middle term $E$ of such a non-split extension is slope $H_{\lambda}$-(semi)stable if and only if $\mathcal{O}_{X}(3,0)$ does not de(semi)stabilize $E$. We have $\xi:=\xi_{\mathcal{O}_{X}(3,0), E}=$ $(3 / 2) H_{0}-(1 / 2) H_{1}$, and the equation $\xi . H_{\lambda}^{2}(\leq) 0$ reads

$$
\frac{3}{2}\left(-2 \lambda^{2}+6 \lambda-1\right) \quad(\leq) \quad 0
$$

Thus, $E$ is slope stable for all polarizations $H_{\lambda}$ with $\lambda^{*}<\lambda<(3 / 2)-(1 / 2) \sqrt{7}$, properly slope semistable for $H_{(3 / 2)-(1 / 2) \sqrt{7}}$, and not semistable for any polarization $H_{\lambda}$ with $\lambda>(3 / 2)-(1 / 2) \sqrt{7}$.

Remark 1.1.6. This example exhibits an interesting phenomenon. Although our set-up is completely algebro-geometric, we naturally encounter objects which are not readily accessible by algebraic methods. In particular, it becomes clear that in order to completely solve our problem we have to find the right notion of Gieseker semistability w.r.t. an arbitrary Kähler class and to construct moduli spaces for them. As Andrei Teleman informed me, this problem has been raised by Tyurin.

Local definition of no future importance. We will say that a pair $(\mathcal{F}, \mathcal{E})$, consisting of a torsion free coherent sheaf $\mathcal{E}$ and a saturated non-zero proper subsheaf $\mathcal{F}$, satisfies the condition (*), if

1. $[\mathcal{E}] \in \mathfrak{F}(\Delta)$,

2. there exists a polarization $H \in \Delta$ such that

(a) $\mu_{H}(\mathcal{F})=\mu_{H}(\mathcal{E})$, and

(b)

$$
\begin{aligned}
& \quad\left((\operatorname{rk} \mathcal{F}-1) c_{1}^{2} \mathcal{F}-2 \operatorname{rk} \mathcal{F} c_{2} \mathcal{F}\right) \cdot H^{n-2} \leq 0 \\
& \text { and }\left((\operatorname{rk} \mathcal{G}-1) c_{1}^{2} \mathcal{G}-2 \operatorname{rk} \mathcal{G} c_{2} \mathcal{G}\right) \cdot H^{n-2} \leq 0, \quad \mathcal{G}:=\mathcal{E} / \mathcal{F}
\end{aligned}
$$

Lemma 1.1.7. $W^{1}:=\left\{x \in(1 / r !) \operatorname{Num}(X) \mid \exists(\mathcal{F}, \mathcal{E})\right.$ satisfying $\left.(*): x=\xi_{\mathcal{F}, \mathcal{E}}\right\}$ is a finite set.

Proof. This is an easy adaptation of the proof of Thm. 1.3 in [7]: Let $x$ be in $W^{1}$. Choose a pair $(\mathcal{F}, \mathcal{E})$ satisfying $(*)$ with $x=\xi_{\mathcal{F}, \mathcal{E}}$. Define

$$
h:=\max \{(s-1) / 2 s+(r-s-1) /(2(r-s)) \mid s=1, \ldots, r\}, \quad l:=(r-1) /(2 r),
$$




$$
k_{1}:=\max \left\{c_{2} \cdot H^{n-2} \mid H \in \Delta\right\}, \quad k_{2}:=\min \left\{c_{1}^{2} \cdot H^{n-2} \mid H \in \Delta\right\} .
$$

Then exactly as in [7], p. 105, one shows that

$$
0 \leq-x^{2} \cdot H^{n-2} \leq r^{2}\left(k_{1}-l k_{2}\right) /(1-h)=: N .
$$

Observe that $N$ depends only on $r, c_{1}$, and $c_{2}$. So, it suffices to show that

$$
\left\{x \in(1 / r !) \operatorname{Num}(X) \mid \exists H \in \Delta: \quad x \cdot H^{n-1}=0 \wedge \quad-x^{2} \cdot H^{n-2} \leq N\right\}
$$

is a finite set. Again, this can be proved in the same manner as Lemma 1.5 in [7]. Indeed, the bilinear form $\langle., .\rangle_{H}$ with $\langle x, y\rangle_{H}=x . y \cdot H^{n-2}$ depends continuously on $H$, and, since $H$ is supposed to be a Kähler class, it has signature $(1, \rho(X)-1)$, by the Hodge-Riemann bilinear relations ([4], p. 123).

\subsection{A boundedness result}

The basis of our investigations is the following

Proposition 1.2.1. The set $\mathfrak{F}(\Delta)$ is bounded.

Proof. Denote by $W^{1 *}$ the set of elements $x \in W^{1}$ such that $x \cdot H^{n-1}=0$ for only finitely many polarizations $H \in \Delta$. For each such $x$, let $w^{1}(x)$ be the set of $H$ such that $x . H^{n-1}$ is zero. We set $w^{1}:=\bigcup_{x \in W^{1 *}} w^{1}(x)$. Let $[\mathcal{E}]$ be in $\mathfrak{F}(\Delta)$, such that $\mathcal{E}$ is slope $H_{\lambda_{0}}$-semistable, $\lambda_{0} \in \mathbb{Q}$, but fails to fulfill the assumptions of Proposition 1.1.3. Then it is easy to check that $H_{\lambda_{0}}$ lies in $w^{1}$. Let $U_{1}, \ldots, U_{s}$ be the connected components of $\Delta \backslash w^{1}$. Pick polarizations $A_{i} \in U_{i} \cap N_{\mathbb{Q}}^{1}(X)$, $i=1, \ldots, s$, and denote by $A_{s+1}, \ldots, A_{t}$ those elements in $w^{1}$ which are rational. By Proposition 1.1.3, the concept of slope (semi)stability remains constant within each $U_{i}$. So, any $\mathcal{E}$ with $[\mathcal{E}] \in \mathfrak{F}(\Delta)$ will be slope semistable w.r.t. one of the polarizations $A_{1}, \ldots, A_{t}$.

\section{Passing through a rational wall}

\subsection{Riemann-Roch}

For any torsion free coherent sheaf $\mathcal{E}$ on $X$, we have its Chern character $\operatorname{ch}(\mathcal{E}) \in$ $A^{*}(X)$. We will denote its homogeneous component of degree $d$ by $\operatorname{ch}_{d}(\mathcal{E})$. We denote by $\operatorname{td}_{e}$ the degree $e$ part of the Todd character of the tangent bundle of $X$. Then, the Riemann-Roch theorem asserts

$$
\chi(\mathcal{E})=\sum_{i=0}^{n} \operatorname{ch}_{i}(\mathcal{E}) \cdot \operatorname{td}_{n-i} .
$$


For any line bundle $\mathcal{L}$ on $X$, we know that $\operatorname{ch}(\mathcal{E} \otimes \mathcal{L})=\operatorname{ch}(\mathcal{E}) \cdot \operatorname{ch}(\mathcal{L})$ so that

$$
\chi(\mathcal{E} \otimes \mathcal{L})=\frac{1}{n !} r \mathcal{L}^{n}+\frac{1}{(n-1) !} \mathcal{L}^{n-1} \cdot\left(\operatorname{ch}_{1}(\mathcal{E})+r \operatorname{td}_{1}\right)+\cdots+\chi(\mathcal{E}) .
$$

In particular, the Hilbert polynomial of $\mathcal{E}$ w.r.t. the ample line bundle $H$ is

$P_{H}(\mathcal{E})=\left(\frac{1}{n !} r H^{n}\right) x^{n}+\left(\frac{1}{(n-1) !} H^{n-1} \cdot\left(\operatorname{ch}_{1}(\mathcal{E})+r \operatorname{td}_{1}\right)\right) x^{n-1}+\cdots+\chi(\mathcal{E})$.

Define $\operatorname{hilb}_{d}(\mathcal{E}):=\operatorname{ch}_{d}(\mathcal{E})+\operatorname{ch}_{d-1}(\mathcal{E}) \cdot \operatorname{td}_{1}+\cdots+r \operatorname{td}_{r}$ for $d=1, \ldots, n$. To abbreviate notation, for a subsheaf $\mathcal{F} \subset \mathcal{E}$ and $0 \leq d \leq n$, we define

$$
\operatorname{hilb}_{d}(\mathcal{F}, \mathcal{E}) \quad:=\frac{\operatorname{hilb}_{d}(\mathcal{E})}{\operatorname{rk} \mathcal{E}}-\frac{\operatorname{hilb}_{d}(\mathcal{F})}{\operatorname{rk} \mathcal{F}}
$$

\subsection{More walls}

We have already defined a set of walls $w^{1}$, such that the concept of slope (semi)stability remains constant between these walls. Define $w^{2}$ as follows: The set of isomorphy classes of sheaves $\mathcal{F}$ which are saturated subsheaves of sheaves in the family $\mathfrak{F}(\Delta)$, such that $\left[\left(c_{1} \mathcal{F} / \mathrm{rk} \mathcal{F}\right)-\left(c_{1} / r\right)\right] \cdot H^{n-1}=0$ for all polarizations in $\Delta$ is bounded, so that they provide us with a finite set of equations $\operatorname{hilb}_{i}(\mathcal{F}, \mathcal{E}) \cdot H^{n-i}=0$. We consider only those equations which are non-trivial and let $w^{2}$ be set of the respective solutions. Set $w:=w^{1} \cup w^{2}$. By the very definition of $w$, the concept of Gieseker (semi)stability remains constant within each connected component of $\Delta \backslash w$.

Remark 2.2.1. i) The walls in $w \backslash w^{1}$ do not affect the concept of slope stability, i.e., the moduli spaces for two polarizations separated only by a wall in $w \backslash w^{1}$ will be isomorphic at least over the open subsets parametrizing slope stable sheaves.

ii) As we have seen in Example 1.1.5, it is possible that $w$ contains points which do not lie in $N_{\mathbb{Q}}^{1}(X)$. In this case the methods presented in this section break down and have only the weak results of Section 3. However, the reader may check that on some simple manifolds such as $\mathbb{P}_{1} \times \mathbb{P}_{n}$, all the walls are rational. In those cases, our results completely describe the situation, at least from an abstract viewpoint. The phenomenon of real walls might explain the difficulties encountered by Qin in the definition of walls for higher dimensional varieties [9].

\subsection{The crucial lemma}

Suppose that $H_{0}$ and $H_{1}$ lie in neighbouring connected components of $\Delta \backslash w$ which are separated by a rational polarization $A$. We can furthermore assume that there is an effective $\mathbb{Q}$-divisor $D$ such that $H_{1}=A+D$ and $H_{0}=A-D$. If $X$ is a surface, 
then in both [7] and [3] the result is based on the fact that there is an integer $l_{0}$ such that $\mathcal{E}$ is Gieseker $H_{1^{-}}\left(H_{0^{-}}\right)\left(\right.$semi)stable if and only if $\mathcal{E}\left(l_{0} D\right)\left(\mathcal{E}\left(-l_{0} D\right)\right)$ is Gieseker $A$-(semi)stable. This result allows one to explore some parameter dependent (semi)stability concept w.r.t. the polarization $A$ such that for different choices of the parameter one obtains $\mathcal{M}_{H_{0}}, \mathcal{M}_{H_{1}}$, and $\mathcal{M}_{A}$, respectively. Now, this choice of parameter corresponds in a suitable construction to the choice of a linearization of a group action. The variation of the quotients in the latter setting is well understood. Indeed, this problem can be appropriately dealt with in the context of master spaces. In the abstract GIT setting, the construction of master spaces is carried out in [11]. Examples of master spaces which solve moduli problems can be found in [8] and [10].

Lemma 2.3.1. There is an integer $l_{0}$ such that for every $l \geq l_{0}$ and every torsion free coherent sheaf $\mathcal{E}$ with Hilbert form $p$ the following conditions are equivalent. 1. $\mathcal{E}$ is Gieseker $H_{1}$-(semi)stable ( $H_{0}$-(semi)stable).

2. $\mathcal{E}(l D)(\mathcal{E}(-l D))$ is Gieseker A-(semi)stable.

Proof. We will explain the proof for $H_{1}$ in the semistable case. It is our task to compare the Hilbert polynomials $P_{H_{1}}(\mathcal{E})$ and $P_{A}(\mathcal{E}(l D))$. Let $\mathcal{E}$ be a torsion free coherent sheaf with Hilbert form $p$, and let $\mathcal{F} \subset \mathcal{E}$ be a non-zero proper subsheaf. One computes

$$
\begin{aligned}
\delta(\mathcal{F}, \mathcal{E}, l)(m):=\frac{\chi\left(\mathcal{E}(l D) \otimes A^{m}\right)}{r}-\frac{\chi\left(\mathcal{F}(l D) \otimes A^{m}\right)}{\operatorname{rk} \mathcal{F}} & \\
= & B_{n-1} \operatorname{hilb}_{1}(\mathcal{F}, \mathcal{E}) \cdot A^{n-1} m^{n-1} \\
& +\left(B_{n-2}^{1} A^{n-2} \cdot \operatorname{hilb}_{2}(\mathcal{F}, \mathcal{E})+B_{n-2}^{2} l A^{n-2} \cdot D \cdot \operatorname{hilb}_{1}(\mathcal{F}, \mathcal{E})\right) m^{n-2}+ \\
& \vdots \\
& +\left(B_{n-i}^{1} A^{n-i} \cdot \operatorname{hilb}_{i}(\mathcal{F}, \mathcal{E})+\cdots+B_{n-i}^{i} l^{i-1} A^{n-i} \cdot D^{i-1} \cdot \operatorname{hilb}_{1}(\mathcal{F}, \mathcal{E})\right) m^{n-i}+ \\
& \vdots \\
& +B_{0}^{1} \operatorname{hilb}_{n}(\mathcal{F}, \mathcal{E})+\cdots+B_{0}^{n} l^{n} D^{n-1} \cdot \operatorname{hilb}_{1}(\mathcal{F}, \mathcal{E})
\end{aligned}
$$

The $B_{i}^{j}$ are just some positive constants of no importance. The coefficient of $m^{n-i}$ in $\delta(\mathcal{F}, \mathcal{E}, l)$ will be denoted by $\delta_{i}(\mathcal{F}, \mathcal{E}, l)$.

Assume $\mathcal{E}$ is Gieseker $H_{1}$-semistable. First, we know by the $H_{1}$-semistability of $\mathcal{E}$ and our assumptions on the walls that $\mathcal{E}$ is at least slope $A$-semistable. If $\mathcal{F}$ is a non-zero proper subsheaf of $\mathcal{E}$ with $\operatorname{hilb}_{1}(\mathcal{F}, \mathcal{E}) \cdot A^{n-1}>0$, then we see that $\mathcal{F}(l D)$ won't $A$-desemistabilize $\mathcal{E}(l D)$ for any $l$. Thus, we can assume that $\operatorname{hilb}_{1}(\mathcal{F}, \mathcal{E}) \cdot A^{n-1}=0$. But the family of all sheaves $\mathcal{F}$ such that there is a Gieseker $A$-semistable sheaf $\mathcal{E}$ containing $\mathcal{F}$ as a non-zero proper saturated subsheaf and $\operatorname{hilb}_{1}(\mathcal{F}, \mathcal{E}) \cdot A^{n-1}=0$ is bounded. This is important to keep in mind for the rest of 
the proof, because it shows that the number of equations arising in the following is indeed finite, and therefore one can find an $l_{0}$ working for all of them. Now, suppose we have a subsheaf $\mathcal{F}$ of $\mathcal{E}$ such that $\delta_{i}(\mathcal{F}, \mathcal{E}, l)=0$ for $i=1, \ldots, j$. By induction we know that then we must have $\operatorname{hilb}_{i}(\mathcal{F}, \mathcal{E}) \cdot A^{n-i}=0$ for $i=1, \ldots, j$, and $\operatorname{hilb}_{i}(\mathcal{F}, \mathcal{E}) \cdot H_{\lambda}^{n-i}=0$ for $i=1, \ldots, j-1$ and every $H_{\lambda}:=A+\lambda D$ with $\lambda \in[0,1]$. If $\operatorname{hilb}_{i}(\mathcal{F}, \mathcal{E}) \cdot H_{\lambda}^{n-i}=0$ for all $H_{\lambda}, \lambda \in[0,1]$, then obviously $A^{n-\iota} \cdot D^{\iota-i} \cdot \operatorname{hilb}_{i}(\mathcal{F}, \mathcal{E})=$ 0 for $\iota=i, \ldots, n$. Therefore,

$$
\delta_{j+1}(\mathcal{F}, \mathcal{E}, l)=B_{n-j-1}^{1} A^{n-j-1} \cdot \operatorname{hilb}_{j+1}(\mathcal{F}, \mathcal{E})+B_{n-j-1}^{2} l A^{n-j-1} \cdot D \cdot \operatorname{hilb}_{j}(\mathcal{F}, \mathcal{E}) .
$$

If we assume $\operatorname{hilb}_{j}(\mathcal{F}, \mathcal{E}) \cdot H_{1}^{n-j}>0$, then our assumption on the walls implies that $\operatorname{hilb}_{j}(\mathcal{F}, \mathcal{E}) \cdot H_{\lambda}^{n-j}>0$ for all $\lambda \in(0,1]$. One checks, by choosing $\lambda$ very small, that this forces $A^{n-j-1}$.D.hilb $j(\mathcal{F}, \mathcal{E})>0$. But then for large $l, \delta_{j+1}(\mathcal{F}, \mathcal{E}, l)>0$, and we don't have to care about $\mathcal{F}$ any more. If, on the other hand, $\operatorname{hilb}_{j}(\mathcal{F}, \mathcal{E}) . H_{1}^{n-j}=$ 0 , then our assumption on the walls shows that $\operatorname{hilb}_{j}(\mathcal{F}, \mathcal{E}) \cdot H_{\lambda}^{n-j}=0$ for all $\lambda \in[0,1]$. The $H$-semistability of $\mathcal{E}$ implies in this case $\operatorname{hilb}_{j+1}(\mathcal{F}, \mathcal{E}) \cdot H_{1}^{n-j-1} \geq 0$. Again using the assumption on the walls, we will also have hilb $b_{j+1}(\mathcal{F}, \mathcal{E}) \cdot A^{n-j-1} \geq$ 0 . In the present circumstances $\operatorname{hilb}_{j+1}(\mathcal{F}, \mathcal{E}) \cdot A^{n-j-1}>(=) 0$ is equivalent to $\delta_{j+1}(\mathcal{F}, \mathcal{E}, l)>(=) 0$. Either we can stop, or we go on with our induction.

Now, let $\mathcal{E}(l D)$ be $A$-semistable for all $l$ sufficiently large. First of all, we remark that this implies that $\mathcal{E}$ is slope $A$-semistable. For any subsheaf $\mathcal{F} \subset \mathcal{E}$ with hilb $1(\mathcal{F}, \mathcal{E}) \cdot A^{n-1}>0$, we will also have hilb $(\mathcal{F}, \mathcal{E}) \cdot H_{1}^{n-1}>0$. Hence, only the saturated subsheaves with $\operatorname{hilb}_{1}(\mathcal{F}, \mathcal{E}) \cdot A^{n-1}=0$ are of interest. But these sheaves live again in a bounded family. Suppose we have a subsheaf $\mathcal{F} \subset \mathcal{E}$ such that $\operatorname{hilb}_{i}(\mathcal{F}, \mathcal{E}) \cdot H_{1}^{n-i}=0$ for $i=1, \ldots, j-1(j=1$ is allowed). Then, of course, $\operatorname{hilb}_{i}(\mathcal{F}, \mathcal{E}) \cdot H_{\lambda}^{n-i}=0$ for $i=1, \ldots, j-1$ and every $\lambda \in[0,1]$. Moreover, $\delta_{i}(\mathcal{F}, \mathcal{E}, l)=$ 0 for $i=1, \ldots, j-1$ in this case, and $\delta_{j}(\mathcal{F}, \mathcal{E}, l)=B_{n-j}^{1} A^{n-j} \cdot \operatorname{hilb}_{j}(\mathcal{F}, \mathcal{E})$. Again, $\delta_{j}(\mathcal{F}, \mathcal{E}, l)>0$ implies $H_{1}^{n-j} \cdot \operatorname{hilb}_{j}(\mathcal{F}, \mathcal{E})>0$, so only the case $\delta_{j}(\mathcal{F}, \mathcal{E}, l)=0$ matters. If $j=n$, we get $(\chi(\mathcal{E}) / r)-(\chi(\mathcal{F}) / \mathrm{rk} \mathcal{F})=0$, whence $\mathcal{F}$ does not $H_{1}$-desemistabilize $\mathcal{E}$. Otherwise, we look at

$$
\delta_{j+1}(\mathcal{F}, \mathcal{E}, l)=B_{n-j-1}^{1} A^{n-j-1} \operatorname{hilb}_{j+1}(\mathcal{F}, \mathcal{E})+l B_{n-j-1}^{2} A^{n-j-1} \cdot D \cdot \operatorname{hilb}_{j}(\mathcal{F}, \mathcal{E}) .
$$

If $H_{1}^{n-j-1} \cdot \operatorname{hilb}_{j}(\mathcal{F}, \mathcal{E})<0$, then $H_{\lambda}^{n-j-1} \cdot \operatorname{hilb}_{j}(\mathcal{F}, \mathcal{E})<0$ for all $\lambda \in(0,1]$. For small $\lambda$ this means $A^{n-j-1}$.D.hilb $j(\mathcal{F}, \mathcal{E})<0$. In this case $\delta_{j+1}(\mathcal{F}, \mathcal{E}, l)<0$ for large $l$, contradicting our assumptions on $\mathcal{E}$.

\subsection{Flips between moduli spaces of parabolic sheaves}

As for $\operatorname{dim} X=1$ [11], one can describe the variation of moduli spaces of parabolic sheaves in terms of GIT flips. Furthermore, they can be flipped to the corresponding Gieseker moduli space. This will be worked out in the present section. 
Parabolic sheaves. Let $X$ be as before, let $A$ be an ample line bundle on $X$, and $D \subset X$ an effective divisor. Fix polynomials $P, P_{1}, \ldots, P_{k}$. Let $\underline{\alpha}=\left(\alpha_{0}, \ldots, \alpha_{k}\right)$ be a weight vector with rational entries $0<\alpha_{0}<\cdots<\alpha_{k}<1$. A parabolic sheaf of weight $\underline{\alpha}$ is a filtration $\mathcal{E}=\mathcal{F}_{0} \supset \mathcal{F}_{1} \supset \cdots \supset \mathcal{F}_{k} \supset \mathcal{F}_{k+1}=\mathcal{E}(-D)$. To shorten notation, we just denote it by $\mathcal{E}$. Define its (parabolic) Hilbert polynomial as $P \frac{\alpha}{A}(\mathcal{E}):=P_{A}(\mathcal{E})-\sum_{i=1}^{k+1} \varepsilon_{i} P_{A}\left(\mathcal{E} / \mathcal{F}_{i}\right)$, where $\varepsilon_{i}:=\alpha_{i}-\alpha_{i-1}, i=0, \ldots, k$, $\alpha_{k+1}:=1$. Given a parabolic sheaf $\mathcal{E}$ of weight $\underline{\alpha}$, every subsheaf $\mathcal{F}$ of $\mathcal{E}$ can be viewed as a parabolic sheaf of weight $\underline{\alpha}$. We say that a parabolic sheaf of weight $\underline{\alpha}$ is (semi)stable if for every non-zero proper subsheaf $\mathcal{F}$ the condition $P_{A}^{\underline{\alpha}}(\mathcal{F}) / \mathrm{rk} \mathcal{F}(\leq) P \frac{\alpha}{A}(\mathcal{E}) / \mathrm{rk} \mathcal{E}$ holds. Of course, one can also define the parabolic slope $\mu \frac{\alpha}{A}$ of $\mathcal{E}$ and speak of slope semistability.

We restrict our attention to parabolic sheaves $\mathcal{E}=\mathcal{F}_{0} \supset \mathcal{F}_{1} \supset \cdots \supset \mathcal{F}_{k} \supset$ $\mathcal{F}_{k+1}=\mathcal{E}(-D)$ of weight $\underline{\alpha}$ where $P_{A}(\mathcal{E})=P$ and $P\left(\mathcal{E} / \mathcal{F}_{i}\right)=P_{i}, i=1, \ldots, k$. The moduli space for S-equivalence classes of semistable parabolic sheaves of weight $\underline{\alpha}$ was constructed in [6] and [12]. Let us denote it by $\mathcal{M}_{A}^{\mathrm{par}}\left(P, P_{1}, \ldots, P_{k} ; \underline{\alpha}\right)$. Below, we will briefly review the construction.

Theorem 2.4.1. Let $P, P_{1}, \ldots, P_{k}$ be as before. Suppose we are given two weight vectors $\underline{\alpha}=\left(\alpha_{0}, \ldots, \alpha_{k}\right)$ and $\underline{\alpha}^{\prime}=\left(\alpha_{0}^{\prime}, \ldots, \alpha_{k}^{\prime}\right)$, and let $\mathcal{M}_{A}(P)$ be the moduli space of S-equivalence classes of Gieseker A-semistable torsion free coherent sheaves with Hilbert polynomial $P$.

Then the spaces $\mathcal{M}_{A}(P), \mathcal{M}_{A}^{\mathrm{par}}\left(P, P_{1}, \ldots, P_{k} ; \underline{\alpha}\right)$, and $\mathcal{M}_{A}^{\mathrm{par}}\left(P, P_{1}, \ldots, P_{k} ; \underline{\alpha}^{\prime}\right)$ can be all constructed via GIT out of the same quasi-projective scheme, i.e., there exists a quasi-projective scheme $\mathfrak{X}$ with an ample line bundle $\mathfrak{L}$ on it, a natural $\mathbb{C}^{* k+1}$-action, and there are linearizations $\sigma_{0}, \sigma$, and $\sigma^{\prime}$ of this $\mathbb{C}^{* k+1}$-action in $\mathfrak{L}$ such that

$$
\begin{aligned}
\mathfrak{X} / / \sigma_{0} \mathbb{C}^{* k+1} & =\mathcal{M}_{A}(P), \\
\mathfrak{X} / / \sigma \mathbb{C}^{* k+1} & =\mathcal{M}_{A}^{\mathrm{par}}\left(P, P_{1}, \ldots, P_{k} ; \underline{\alpha}\right), \\
\mathfrak{X} / / \sigma^{\prime} \mathbb{C}^{* k+1} & =\mathcal{M}_{A}^{\mathrm{par}}\left(P, P_{1}, \ldots, P_{k} ; \underline{\alpha}^{\prime}\right) .
\end{aligned}
$$

Thus, by the Mumford-Thaddeus principle ([11], [8], Part 1), these spaces are related by a sequence of $\mathbb{C}^{* k+1}$-flips.

Some useful semistability criteria. Let $W_{0}, \ldots, W_{k}$ be finite dimensional $\mathbb{C}$-vector spaces. Define $W:=W_{0} \oplus \cdots \oplus W_{k}$, and let $\mathbb{C}^{* k}$ act on $W$ in the following way: The $i$-th factor of $\mathbb{C}^{* k}$ acts by scalar multiplication on $W_{i}$ and trivially on all other summands, $i=1, \ldots, k$. In this way, we obtain a linearized action of $\mathbb{C}^{* k}$ on $\mathbb{P}(W)$. By means of an induction, one derives the following observation from [8], Example 1.2.5.

Lemma 2.4.2. Considering all possible linearizations of the above $\mathbb{C}^{* k}$-action on 
$\mathbb{P}(W)$, one obtains the following polarized quotients

$$
\left(\left(\mathbb{P}\left(W_{\iota_{1}}\right) \times \cdots \times \mathbb{P}\left(W_{\iota_{\kappa}}\right)\right),\left[\mathcal{O}\left(a_{1}, \ldots, a_{\kappa}\right)\right]\right) .
$$

Here, $\left\{\iota_{1}, \ldots, \iota_{\kappa}\right\}$ can be any subset of $\{0, \ldots, k\}$, and $\left(a_{1}, \ldots, a_{\kappa}\right)$ any tuple of positive integers.

Consider a reductive algebraic group $G$ and representations $\rho_{i}: G \longrightarrow \operatorname{GL}\left(W_{i}\right)$, $i=0, \ldots, k$. The direct sum of these representations defines an $\mathcal{O}_{\mathbb{P}(W)}(1)$-linearized action of $G$ on $\mathbb{P}(W)$. We also have $\mathcal{O}_{\mathbb{P}\left(W_{i}\right)}$ (1)-linearized actions of $G$ on $\mathbb{P}\left(W_{i}\right)$, $i=0, \ldots, k$, and for a point $\left[v_{i}\right] \in \mathbb{P}\left(W_{i}\right)$ and a one parameter subgroup $\lambda: \mathbb{C}^{*} \longrightarrow G$ we let $\mu_{i}\left(\left[v_{i}\right], \lambda\right)$ be minus the weight of the induced $\mathbb{C}^{*}$-action on the fibre of $\mathcal{O}_{\mathbb{P}\left(W_{i}\right)}(1)$ over the point $\lim _{z \longrightarrow \infty} \lambda(z) \cdot\left[v_{i}\right]$.

Proposition 2.4.3. Let $w=\left[v_{0}, \ldots, v_{k}\right] \in \mathbb{P}(W)$ be a point, and let $\left(\nu_{1}, \ldots, \nu_{\mu}\right)$ be the indices with $v_{\nu_{j}} \neq 0, j=1, \ldots, \mu$. Then the following conditions are equivalent: 1. $w$ is $G$-semistable w.r.t. given linearization.

2. There exist non-negative integers $l_{\nu_{1}}, \ldots, l_{\nu_{\mu}}$, not all zero, such that for any one parameter subgroup $\lambda: \mathbb{C}^{*} \longrightarrow G$

$$
l_{\nu_{1}} \mu_{\nu_{1}}\left(\left[v_{\nu_{1}}\right], \lambda\right)+\cdots+l_{\nu_{\mu}} \mu_{\nu_{\mu}}\left(\left[v_{\nu_{\mu}}\right], \lambda\right) \geq 0
$$

Remark 2.4.4. In view of Lemma 2.4.2, the second condition means that we find a linearization of the $\mathbb{C}^{* k}$-action such that the image of $w$ in the corresponding polarized quotient is $G$-semistable w.r.t. the induced linearization.

Proof. We observe that the hypothesis that $G$ have no characters in Section 1.2. of [8] only assures that the linearization of $G$ is unique. In the proofs, this assumptions is never used. So, we can apply [8], Thm. 1.4.1, to prove the assertion by induction. The details are left to the reader.

A "baby" master space construction. In this section, we explain the proof of Theorem 2.4.1. To avoid excessive indices and formulas, we will only treat the case $k=0$ which is the only one we will need for our applications. Using the semistability criteria given above, the reader will have no difficulty to extend the proof to the case of arbitrary $k$. We need to fix a Poincaré sheaf $\mathfrak{P}$ on Pic $X \times X$.

First of all, we may choose an integer $m_{0}$ such that for every $m \geq m_{0}$ and every torsion free coherent sheaf $\mathcal{E}$ which is either slope $A$-semistable or which appears in a parabolic sheaf of either weight $\underline{\alpha}$ or $\underline{\alpha}^{\prime}$

- $H^{i}(X, \mathcal{E}(m A))=0$ for $i=1, \ldots, n$.

- $\mathcal{E}(m A)$ is generated by global sections.

- The same holds for $\mathcal{E}_{\mid D}(m A)$. 
Moreover, let $\mathfrak{A} \subset \operatorname{Pic} X$ be the union of all components containing elements of the form $[\operatorname{det} \mathcal{E}]$.

- Then $\mathcal{L}(r m A)$ is globally generated and without higher cohomology for every $[\mathcal{L}] \in \mathfrak{A}$.

As usual, we consider the Quot scheme $\mathfrak{F}$ of equivalence classes of quotients $q: V \otimes$ $\mathcal{O}_{X}(-m A) \longrightarrow \mathcal{E}$ where $\mathcal{E}$ is a coherent $\mathcal{O}_{X}$-module with Hilbert polynomial $P$. Furthermore, there is a universal flag

$$
V \otimes \pi_{X}^{*} \mathcal{O}_{X}(-m A) \longrightarrow \mathfrak{E}_{\mathfrak{F}} \longrightarrow \mathfrak{E}_{\mathfrak{F} \mid \mathfrak{F} \times D}
$$

over $\mathfrak{F} \times X$. Let $U_{0}$ be the set of points $\left[q: V \otimes \mathcal{O}_{X}(-m A) \longrightarrow \mathcal{E} \longrightarrow \mathcal{E}_{\mid D}\right]$ for which $\mathcal{E}$ is Gieseker $A$-semistable, let $U_{\underline{\alpha}}$ and $U_{\underline{\alpha}^{\prime}}$ be the sets for which $\mathcal{E} \supset \mathcal{E}(-D)$ is a semistable parabolic sheaf of weight $\underline{\alpha}$ and $\underline{\alpha}^{\prime}$, resp., and $U:=U_{0} \cup U_{\underline{\alpha}} \cup U_{\underline{\alpha}^{\prime}}$. The sheaf $\pi_{\mathfrak{F} *}\left(\mathfrak{E}_{\mathfrak{F}} \otimes \pi_{X}^{*} \mathcal{O}_{X}(m A)\right)$ is locally free of rank $P(m)$, and the sheaf $\pi_{\mathfrak{F} *}\left(\mathfrak{E}_{\mathfrak{F} \mid \mathfrak{F} \times D} \otimes \pi_{X}^{*} \mathcal{O}_{X}(m A)\right)$ is locally free of rank, say, $R$. The scheme $U$ can the be mapped $\mathrm{SL}(V)$-equivariantly to

$$
\mathbb{P}\left(\underline{\operatorname{Hom}}\left(\bigwedge^{r} V \otimes \mathcal{O}_{\mathfrak{A}}, \pi_{\mathfrak{A} *}\left(\mathfrak{P} \otimes \pi_{X}^{*} \mathcal{O}_{X}(m A)\right)^{\vee}\right) \times \mathbb{P}\left(\bigwedge^{R}\left(V \otimes H^{0}\left(\mathcal{O}_{X}(m A)\right)\right)^{\vee}\right)\right.
$$

Let $\mathbb{P}_{\mathfrak{A}}$ be the first factor of this product, and $\mathbb{P}_{R}$ the second. Choose some ample sheaf $\mathfrak{H}_{\mathfrak{A}}$ on $\mathfrak{A}$, so that $\mathfrak{L}_{\mathfrak{A}}:=\mathcal{O}_{\mathbb{P}_{\mathfrak{A}}}(1) \otimes \pi_{\mathfrak{A}}^{*} \mathfrak{H}_{\mathfrak{A}}$ is ample. The sheaf $\pi_{\mathbb{P}_{\mathfrak{A}}}^{*} \mathfrak{L}_{\mathfrak{A}}^{\otimes a} \otimes$ $\pi_{\mathbb{P}_{R}}^{*} \mathcal{O}_{\mathbb{P}_{R}}(b)$ on $\mathbb{P}_{\mathfrak{A}} \times \mathbb{P}_{R}$ will be denoted by $\mathcal{O}(a, b)$. Denote by $U_{0}^{\prime}$ the set of $\operatorname{SL}(V)$ semistable points w.r.t. the linearization in $\mathcal{O}(1,0)$. Then $U_{0}$ is mapped injectively and properly to $U_{0}^{\prime}$, and for suitable choices of $(a, b)$ and $\left(a^{\prime}, b^{\prime}\right)$, the sets $U_{\underline{\alpha}}$ and $U_{\underline{\alpha}^{\prime}}$ get immersed into the sets $U_{\underline{\alpha}}^{\prime}$ and $U_{\underline{\alpha}^{\prime}}^{\prime}$ of points which are $\mathrm{SL}(V)$-semistable w.r.t. the linearization in $\mathcal{O}(a, b)$ and $\mathcal{O}\left(a^{\prime}, b^{\prime}\right)$, respectively. Altogether, we obtain an injective and proper map of $U$ to $U^{\prime}:=U_{0}^{\prime} \cup U_{\underline{\alpha}}^{\prime} \cup U_{\underline{\alpha}^{\prime}}^{\prime}$. It is now clear that the moduli spaces we are interested in are obtained from $\bar{U}$ by dividing out $\mathrm{SL}(V)$ for different linearizations. To understand the assertion about the $\mathbb{C}^{*}$-flips, we proceed as follows. Define $\mathfrak{R}$ as the projective bundle over $\mathfrak{Q}$ associated to the vector bundle

$\pi_{\mathfrak{F} * \underline{\operatorname{Hom}}}\left(\operatorname{det}\left(\mathfrak{E}_{\mathfrak{F}} \otimes \pi_{X}^{*} \mathcal{O}_{X}(m A)\right),\left(\operatorname{det} \times \operatorname{id}_{X}\right)^{*} \mathfrak{P}\right) \oplus \bigwedge^{R}\left(V \otimes H^{0}\left(\mathcal{O}_{X}(m A)\right)\right) \otimes \mathcal{O}_{\mathfrak{F}}$

$\operatorname{det}: \mathfrak{F} \longrightarrow \mathfrak{A}$ being associated with the family $\mathfrak{E}_{\mathfrak{F}}$, and $\mathfrak{S}$ the projective bundle

$$
\mathbb{P}\left(\underline{\operatorname{Hom}}\left(\bigwedge^{r} V \otimes \mathcal{O}_{\mathfrak{A}}, \pi_{\mathfrak{A} *}\left(\mathfrak{P} \otimes \pi_{X}^{*} \mathcal{O}_{X}(m A)\right)\right)^{\vee} \oplus \bigwedge^{R}\left(V \otimes H^{0}\left(\mathcal{O}_{X}(m A)\right)\right)^{\vee} \otimes \mathcal{O}_{\mathfrak{A}}\right)
$$

over $\mathfrak{A}$. One has the natural morphism $\mathfrak{t}: \mathfrak{R} \longrightarrow \mathfrak{S}$ (compare [8], Section 2.4). There are natural $\left(\operatorname{SL}(V) \times \mathbb{C}^{*}\right)$-actions on $\mathfrak{R}$ and $\mathfrak{S}$, and $\mathfrak{t}$ is equivariant. The 
$\mathrm{SL}(V)$-action is canonically linearized, and we can choose linearizations $s_{0}, s$, and $s_{1}$ of the $\mathbb{C}^{*}$-action such that the polarized quotients are

$$
\begin{aligned}
\mathfrak{S} / / s_{0} \mathbb{C}^{*} & =\left(\mathbb{P}_{\mathfrak{A}},\left[\mathfrak{L}_{\mathfrak{A}}\right]\right) \\
\mathfrak{S} / / \mathbb{C}^{*} & =\left(\mathbb{P}_{\mathfrak{A}} \times \mathbb{P}_{R},[\mathcal{O}(a, b)]\right) ; \\
\mathfrak{S} / / s_{s^{\prime}} \mathbb{C}^{*} & =\left(\mathbb{P}_{\mathfrak{A}} \times \mathbb{P}_{R},\left[\mathcal{O}\left(a^{\prime}, b^{\prime}\right)\right]\right) .
\end{aligned}
$$

Let $U_{0}^{\prime \prime \prime}, U_{\underline{\alpha}}^{\prime \prime \prime}$, and $U_{\underline{\alpha}^{\prime}}^{\prime \prime \prime}$ be the respective sets of $\left(\operatorname{SL}(V) \times \mathbb{C}^{*}\right)$-semistable points, and let $U^{\prime \prime \prime}$ be their union. Their preimages $U_{0}^{\prime \prime}, U_{\alpha}^{\prime \prime}$, and $U_{\alpha^{\prime}}^{\prime \prime}$ under $\mathfrak{t}$ coincide with the preimages of $U_{0}, U_{\alpha}$, and $U_{\alpha^{\prime}}$ under the bundle map $\mathfrak{R}^{\underline{\prime}} \longrightarrow \mathfrak{F}$. Thus, the union $U^{\prime \prime}$ of these sets maps finitely to $U^{\prime \prime \prime}$. By general properties of good quotients, the quotient $\mathfrak{Y}:=U^{\prime \prime \prime} / / \mathrm{SL}(V)$ is an open subset of the projective scheme $\mathfrak{S} / / \mathrm{SL}(V)$, and $\mathfrak{X}:=U^{\prime \prime} / / \mathrm{SL}(V)$ maps finitely to $\mathfrak{Y}$; call the corresponding map $\mathfrak{z}$. Both, $\mathfrak{X}$ and $\mathfrak{Y}$ inherit $\mathbb{C}^{*}$-actions, and $\mathfrak{z}$ is equivariant w.r.t. them. By construction and the "commutation principle" (e.g., [8], Sect. 1.3.1), the $\mathbb{C}^{*}$-action on $\mathfrak{Y}$ is linearized in an ample line bundle $\mathfrak{L}_{\mathfrak{Y}}$ such that suitable manipulations of this linearization will yield $\mathfrak{S} / / s_{0}\left(\mathrm{SL}(V) \times \mathbb{C}^{*}\right)$ and so on as quotients. Pulling back these linearizations to $\mathfrak{X}$ gives us $\mathfrak{L}, \sigma_{0}, \sigma$, and $\sigma^{\prime}$ as asserted.

\subsection{The proof of the Main Theorem}

We return to the setting of Section 2.3 and choose some $l$ for which Lemma 2.3.1 holds. For a torsion free coherent sheaf $\mathcal{E}$ and $\beta \in[0,1]$, we set $P_{A}^{\beta}(\mathcal{E}):=(1-$ $\beta) P_{A}(\mathcal{E}(-l D))+\beta P_{A}(\mathcal{E}(l D))$, and call $\mathcal{E} \beta$-(semi) stable, if and only if $P_{A}^{\beta}(\mathcal{F}) / \mathrm{rk} \mathcal{F}$ $(\leq) P_{A}^{\beta}(\mathcal{E}) / \mathrm{rk} \mathcal{E}$ for any non-trivial proper subsheaf $\mathcal{F}$. In Lemma 2.3.1, we have seen that a torsion free coherent sheaf $\mathcal{E}$ with Hilbert form $p$ is $H_{1^{-}}\left(H_{0^{-}}\right)$ (semi)stable if and only if $\mathcal{E}$ is 1-(0-)(semi)stable. But as the proof of Lemma 2.3.1 shows, we can choose $\beta_{1}$ close to one and $\beta_{2}$ close to zero, so that we will also have that $\mathcal{E}$ is $H_{1^{-}}\left(H_{0^{-}}\right)$(semi)stable if and only if $\mathcal{E}$ is $\beta_{1^{-}}\left(\beta_{0^{-}}\right)$(semi)stable. As a corollary to the existence of moduli of parabolic bundles (the rôle of $\mathcal{E}$ is the last section will now be played by $\mathcal{E}(l D)$ and that of $D$ by $2 l D)$, for any $\beta \in(0,1)$, there exists a projective moduli scheme $\mathcal{M}_{A}^{\beta}(p)$ of S-equivalence classes of $\beta$-semistable torsion free coherent sheaves with Hilbert form $p$, and as we have seen in 2.3.1 $\mathcal{M}_{A}^{\beta_{i}}(p) \cong \mathcal{M}_{H_{i}}(p)$, for $i=0,1$. Therefore, the main theorem is a direct consequence of Theorem 2.4.1.

\section{Passing through an arbitrary wall}

Let $H_{0}$ and $H_{1}$ be two polarizations, and $\mathfrak{F}\left(H_{0}\right)$ and $\mathfrak{F}\left(H_{1}\right)$ be the set of isomorphy classes of torsion free coherent sheaves which are slope $H_{0}$-semistable and slope $H_{1}$-semistable, respectively. Let $H$ be an arbitrary polarization and write $\mathcal{O}_{X}(m)$ for $\mathcal{O}_{X}(m H)$. Since both $\mathfrak{F}\left(H_{0}\right)$ and $\mathfrak{F}\left(H_{1}\right)$ are bounded, we can find a complex 
vector space $V$ and an integer $m_{0}$ such that any sheaf $\mathcal{E}$ whose isomorphy class belongs to either $\mathfrak{F}\left(H_{0}\right)$ or $\mathfrak{F}\left(H_{1}\right)$ can be embedded into $V \otimes \mathcal{O}_{X}(m)$ for all $m \geq m_{0}$. We denote by $\mathfrak{Q}$ the Quot scheme of all submodules of $V \otimes \mathcal{O}_{X}\left(m_{0}\right)$ with Hilbert form $p$. Strictly speaking, this is a fine moduli space of $\delta$-stable pairs $(\mathcal{E}, \varphi)$, $\varphi \in \operatorname{Hom}\left(\mathcal{E}, V \otimes \mathcal{O}_{X}\left(m_{0}\right)\right)$, for some large polynomial $\delta$. But as its universal property shows, it is isomorphic to a Quot scheme and, in particular, does not depend on the choice of a polarization.

Fix a Poincaré sheaf $\mathfrak{P}$ on Pic $X \times X$, and let $\mathcal{M}_{H_{i} / \mathfrak{P} / V \otimes \mathcal{O}_{X}\left(m_{0}\right)}(p)$ be the master space of S-equivalence classes of semistable $\mathfrak{P}$-oriented pairs $(\mathcal{E}, \varepsilon, \varphi)[8]$ where $\mathcal{E}$ is a torsion free coherent sheaf with Hilbert polynomial $P_{H_{i}}(n)=p\left(H_{i}^{\otimes n}\right)$, for all $n \in \mathbb{N}, \varepsilon: \operatorname{det} \mathcal{E} \longrightarrow \mathfrak{P}_{\mid\{[\operatorname{det} \mathcal{E}]\} \times X}$ is a homomorphism, and $\varphi \in \operatorname{Hom}(\mathcal{E}, V \otimes$ $\left.\mathcal{O}_{X}\left(m_{0}\right)\right), i=1,2$. As proved in [8], there are natural $\mathbb{C}^{*}$-actions on these master spaces. Suitably linearized, these $\mathbb{C}^{*}$-actions give rise to sequences of $\mathbb{C}^{*}$ flips which begin with a fibration $\pi_{i}: \mathfrak{M}_{i} \longrightarrow \mathcal{M}_{H_{i}}(p)$ and end in $\mathfrak{Q}$. The fibre of $\pi_{i}: \mathfrak{M}_{i} \longrightarrow \mathcal{M}_{H_{i}}(p)$ over the isomorphy class of a stable sheaf $\mathcal{E}$ is just $\mathbb{P}\left(\operatorname{Hom}\left(\mathcal{E}, V \otimes \mathcal{O}_{X}\left(m_{0}\right)\right)^{\vee}\right)$. Therefore, we have shown that the fibrations $\pi_{0}: \mathfrak{M}_{0}$

$\longrightarrow \mathcal{M}_{H_{0}}(p)$ and $\pi_{1}: \mathfrak{M}_{1} \longrightarrow \mathcal{M}_{H_{1}}(p)$ can be created by means of $\mathbb{C}^{*}$-flips out of the Quot scheme $\mathfrak{Q}$.

\section{Acknowledgements}

The paper was inspired by the suggestion of Professor Miró-Roig to study the birational geometry of moduli spaces of sheaves over higher dimensional base varieties. I also profited from discussions with Laura Costa and Manfred Lehn. During the preparation of the article, the author was supported by grant \#1996SGR00060 of the Direcció General de Recerca, Generalitat de Catalunya. Final revisions were made during the author's stay at Bar-Ilan University during which he was funded by the Emmy-Noether Institute.

\section{References}

[1] E. Ballico, R.-M. Miró-Roig, A lower bound for the number of components of the moduli schemes of stable rank 2 vector bundles on projective threefolds, Preprint.

[2] L. Ein, Generalized null correlation bundles, Nagoya Math. J. 111 (1988), 13-24.

[3] G. Ellingsrud, L. Göttsche, Variation of moduli spaces and Donaldson invariants under change of polarization, J. reine angew. Math. 467 (1995), 1-49.

[4] Ph. Griffiths, J. Harris, Principles of Algebraic Geometry, Wiley Interscience, 1978.

[5] D. Huybrechts, M. Lehn, The Geometry of Moduli Spaces of Sheaves, Vieweg, 1997.

[6] M. Maruyama, K. Yokogawa, Moduli of parabolic stable sheaves, Math. Ann. 293 (1992), $77-99$.

[7] K. Matsuki, R. Wentworth, Mumford-Thaddeus principle on the moduli space of vector bundles on an algebraic surface, Int. J. Math. 8 (1997), 97-148.

[8] Ch. Okonek, A. Schmitt, A. Teleman, Master spaces for stable pairs, Topology 38 (1999), 117-39. 
[9] Z. Qin, Equivalence classes of polarizations and moduli spaces of sheaves, J. Diff. Geom. 37 (1993), 397-415.

[10] A. Schmitt, Framed Hitchin pairs, Rev. Roumaine Math. Pures Appl. (to appear).

[11] M. Thaddeus, Geometric invariant theory and flips, J. AMS 9 (1996), 691-725.

[12] K. Yokogawa, Compactification of moduli of parabolic sheaves and moduli of parabolic Higgs sheaves, J. Math. Kyoto Univ. 33 (1993), 451-504.

Alexander Schmitt

Universität GH Essen

FB 6 Mathematik und Informatik

D-45117 Essen

Deutschland

e-mail: alexander.schmitt@uni-essen.de

(Received: January 20, 1998) 